\title{
BMJ Open Physical activity and risk of fatty liver in people with different levels of alcohol consumption: a prospective cohort study
}

\author{
Kenji Tsunoda, ${ }^{1}$ Yuko Kai, ${ }^{1}$ Ken Uchida, ${ }^{2}$ Tsutomu Kuchiki, ${ }^{3}$ Toshiya Nagamatsu ${ }^{1}$
}

To cite: Tsunoda K, Kai Y, Uchida K, et al. Physical activity and risk of fatty liver in people with different levels of alcohol consumption: a prospective cohort study. BMJ Open 2014;4:e005824. doi:10.1136/bmjopen-2014005824

- Prepublication history and additional material is available. To view please visit the journal (http://dx.doi.org/ 10.1136/bmjopen-2014005824).

Received 30 May 2014 Revised 8 July 2014 Accepted 10 July 2014

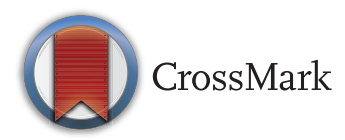

${ }^{1}$ Physical Fitness Research Institute, Meiji Yasuda Life Foundation of Health and Welfare, Hachioji, Tokyo, Japan

${ }^{2}$ Meiji Yasuda Shinjuku Medical Center, Meiji Yasuda Life Foundation of Health and Welfare, Shinjuku, Tokyo, Japan

${ }^{3}$ Meiji Yasuda Wellness Development Office, Meiji Yasuda Life Foundation of Health and Welfare, Shinjuku, Tokyo, Japan

Correspondence to Dr Kenji Tsunoda; tsunoda@my-zaidan.or.jp

\section{ABSTRACT}

Objective: To investigate whether physical activity affects the future incidence of fatty liver in people with never-moderate and heavy alcohol consumption.

Design: Prospective cohort study.

Setting: Health check-up programme at Meiji Yasuda Shinjuku Medical Center in Shinjuku Ward, Tokyo, Japan. Population: A total of 10146 people aged 18 years or older without fatty liver enrolled through baseline surveys conducted from 2005 to 2007. They were grouped into never-moderate alcohol drinkers $(n=7803)$ and heavy alcohol drinkers $(n=2343)$ and followed until 2013.

Main outcome measure: Incident fatty liver diagnosed by ultrasound.

Results: During a mean follow-up of 4.4 years (34 648 person-years), 1255 never-moderate alcohol drinkers developed fatty liver; 520 heavy alcohol drinkers developed fatty liver during a mean follow-up of 4.1 years (9596 person-years). For never-moderate alcohol drinkers, engaging in $>3 \times$ /week of low-intensity ( $\mathrm{HR}=0.82,95 \% \mathrm{Cl} 0.71$ to 0.96 ) and moderate-intensity ( $\mathrm{HR}=0.56,95 \% \mathrm{Cl} 0.39$ to 0.81 ) physical activity significantly reduced incident fatty liver compared with those who engaged in physical activity $<1 \times /$ week. For vigorous-intensity physical activity, frequencies of $2 \times /$ week ( $\mathrm{HR}=0.57,95 \% \mathrm{Cl} 0.38$ to 0.86 ) and $>3 \times /$ week ( $\mathrm{HR}=0.55,95 \% \mathrm{Cl} 0.38$ to 0.79 ) were significantly associated with lower risk of incident fatty liver. In propensity-adjusted models, these significant associations still remained. By contrast, in heavy alcohol drinkers, there were no significant associations between the type or frequency of physical activity and incident fatty liver.

Conclusions: Physical activity had an independent protective effect on incident fatty liver only in the nevermoderate alcohol drinkers, and the preventive effect increased with higher frequencies and intensities of physical activity.

\section{INTRODUCTION}

Alcoholic fatty liver disease (AFLD) is a wellknown hepatic disorder. ${ }^{12}$ However, concern is growing over non-AFLD (NAFLD) because NAFLD, as well as AFLD, can progress to

\section{Strengths and limitations of this study}

- This study revealed the independent preventive effect of physical activity on incident nonalcoholic fatty liver disease; its strength lies in its prospective cohort design.

- Our large sample size allowed us to show separate HRs according to frequencies and intensities of physical activity.

- Although hepatic ultrasonography is widely used at the population level, it can lead to incorrect diagnoses.

hepatitis and fibrosis. ${ }^{3-5}$ The incidence of NAFLD has gradually increased ${ }^{6}$; a recent Japanese cohort study ${ }^{7}$ has reported that $29.7 \%$ of health check-up examinees had NAFLD. Western countries have had a high prevalence of NAFLD for some time, ${ }^{8}$ but recently NAFLD has become an urgent issue for the international community including Japan. $^{6} 89$

Physical activity (PA) is a well-known way of preventing and improving certain obesityrelated diseases such as hypertension, ${ }^{10}$ diabetes ${ }^{11}$ and dyslipidaemia. ${ }^{12}$ Since NAFLD ${ }^{13} 14$ and $\mathrm{AFLD}^{15} 16$ are obesity-related, PA may also have an effect on these diseases. In fact, several cross-sectional $^{17-21}$ and retrospective ${ }^{22}$ studies already revealed a significant association between higher levels of PA and a lower prevalence of NAFLD. However, a prospective association is still unclear, and evidence from a longitudinal cohort design is needed. ${ }^{23}$

Additionally, recent population studies on PA and fatty liver focused on NAFLD and excluded people with a heavy alcohol intake ${ }^{17-22}$; there are few epidemiological findings on the effect of PA on AFLD. Confirming the preventive effect of PA on fatty liver for light and heavy alcohol drinkers is useful information for all people, but especially for those who cannot cut down or stop drinking. 
The purpose of this prospective cohort study was to investigate whether engaging in PA prevents the future incidence of fatty liver diagnosed by ultrasound in two populations: never-moderate alcohol drinkers and heavy alcohol drinkers.

\section{METHODS}

\section{Participants and data collection}

We used data from the Meiji Yasuda Longitudinal Study, a prospective cohort study based on annual health checkups conducted in Meiji Yasuda Shinjuku Medical Center in Shinjuku Ward, Tokyo, Japan. The majority of patients were employees and their spouses, with employers providing financial support for the annual health check-ups. This popular method of providing medical services in Japan is called 'a human dock'. It is also an important source for research participants and data including fatty liver studies. ${ }^{6} 71424$ Figure 1 shows the flow of participants through the study. We used 2005-2007 survey data $(n=25056$, aged 18 years or older) as our baseline data. Of these people, 2541 individuals were excluded due to the lack of an ultrasound confirming their fatty liver and 2365 due to incomplete data. We further excluded 1328 because they had histories of liver disease, including hepatitis B or C, cirrhosis and hepatic haemangioma, they were using drugs associated with hepatic disease or they had antibodies to hepatitis B or C. We excluded 3832 individuals because they had fatty liver disease at the baseline. Furthermore, 4844 individuals were excluded because they could not be followed for at least 1 year. We had a final tally of 10146 participants. These participants were followed through their annual health check-ups until fatty liver disease had been diagnosed or until the end of 2013. When a participant we were following did not attend an annual check-up, we used all available follow-up data. All participants provided informed consent.

\section{Assessment of fatty liver and alcohol consumption}

Abdominal ultrasonography machines (EUB-2000, Hitachi, Japan; and SSA-340, 550, 580 and 660, Toshiba, Japan) were used to diagnose fatty liver based on known standard criteria, including hepatorenal echo contrast, liver brightness, deep attenuation and vascular blurring. ${ }^{25}{ }^{26} \mathrm{~A}$ fatty liver appears bright in ultrasound images compared with the kidney; this is the most frequently observed sign of fatty liver. ${ }^{25}$ In severe fatty liver, deep attenuation and vascular blurring are also frequently observed ${ }^{25}$ To enhance diagnostic accuracy, we evaluated the ultrasound images in three steps: first, a trained medical technologist performed the ultrasound and provided opinions with images to the doctor; second, the doctor made a diagnosis based on this information and third, a group of medical technologists including the original examiner confirmed the doctor's diagnosis. The mean diagnosis rate of fatty liver in our surveys from 2005 to 2013 was $23.1 \pm 1 \%$ (range 22.2$24.8 \%)$. Ultrasound diagnosis of fatty liver has been validated in a systematic review. ${ }^{26}$

Using a self-administered questionnaire, participants revealed their alcohol intake frequency (never, occasional drink, 1-2 days/week, 3-4 days/week, daily with day off drinking and daily without day off drinking) and the quantity of each type of alcoholic beverage consumed. To determine the quantity of alcohol consumed, participants used information provided on the alcohol/ ethanol content of each beverage type equivalent to sake. One go (a traditional Japanese measurement) of sake (23 $\mathrm{g}$ of alcohol) is roughly equivalent to 2 glasses of wine, $633 \mathrm{~mL}$ of beer, 2.5 single glasses of whisky or 0.5 cup of shochu. We used a scoring method for the frequency of alcohol consumption as follows: 0.5 for an occasional drink, 1.5 for 1-2 days/week, 3.5 for 34 days/week, 5.5 for daily with day off drinking and 7 for daily without day off drinking. We set four alcohol categories by calculating the average daily alcohol consumption: never, moderate (less than $23 \mathrm{~g}$ of alcohol/ day), heavy (23-45.9 g/day) and very heavy ( $46 \mathrm{~g} /$ day or more). ${ }^{27}$ The validation for this kind of assessment for alcohol consumption was reported in a previous Japanese cohort study. ${ }^{28}$ Based on the alcohol intake status at the baseline, participants were divided into never-to-moderate alcohol drinkers $(n=7803)$ and heavy alcohol drinkers $(\mathrm{n}=2343) .{ }^{27}$
Figure 1 Flow of eligible participants in this study. †At this stage, 3832 of 18822 examinees $(20.4 \%$ of total, $29.6 \%$ of men, $9.8 \%$ of women) were diagnosed with fatty liver. When looking at examinees' levels of alcohol consumption, 2827 of 14490 never-moderate alcohol drinkers $(19.5 \%$ of total, $31.1 \%$ of men, $10.0 \%$ of women) and 1005 of 4332 heavy alcohol drinkers (23.2\% of total, $26.8 \%$ of men, $7.8 \%$ of women) were diagnosed with fatty liver at the baseline.

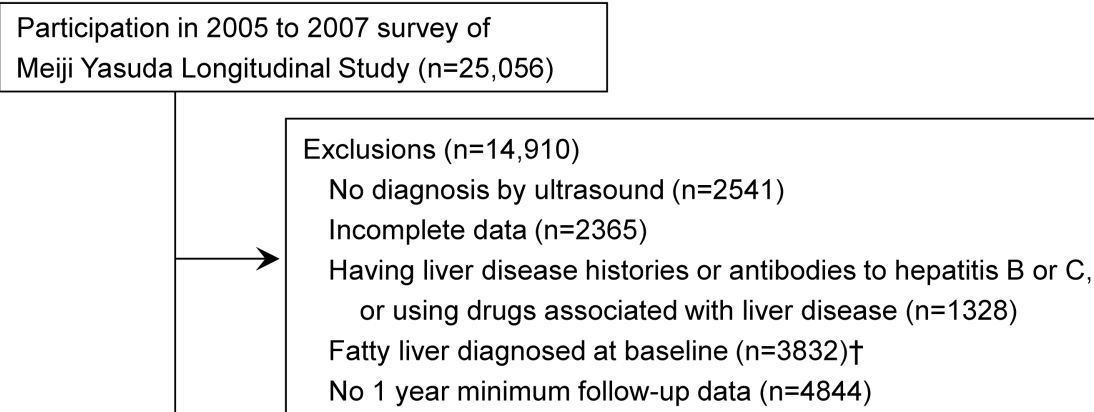

Cases included in analyses $(n=10,146$, mean age $=48.1 \pm 10.7$ years, male $=48.7 \%$ )

Never-moderate alcohol drinkers $(n=7803$, mean age $=47.8 \pm 10.9$ years, male $=39.5 \%)$ Heavy alcohol drinkers $(n=2343$, mean age $=49.1 \pm 9.8$ years, male $=79.6 \%)$ 


\section{Physical activity}

A questionnaire assessed leisure-time $\mathrm{PA}$ in a typical week by frequency (never, $<1 \times, 1 \times, 2 \times$ and $>3 \times /$ week), duration (minutes per session) and intensity (low, moderate, vigorous and very vigorous). Low-intensity PA includes activities such as walking, light bicycling, gymnastics, light dancing, golf and Japanese croquet. A moderate-intensity PA includes jogging, bicycling (about $16 \mathrm{~km} / \mathrm{h}$ ), hiking, badminton, tennis and ballroom dancing. A vigorous-intensity PA includes jogging (about $9.6 \mathrm{~km} / \mathrm{h}$ ), swimming, climbing hills and aerobic dancing. A very vigorous PA includes running a marathon, rope jumping and competitive sports such as soccer and rugby. Because few respondents participated in very vigorous $\mathrm{PA}$, we combined the very vigorous and vigorous PA into a single group of vigorous-intensity PA. The low-intensity activities corresponded to about 3-5 metabolic equivalents (METs), moderate-intensity corresponded to 5-7 METs and vigorous-intensity corresponded to 7 or more METs. ${ }^{29} 30$

Since $10 \mathrm{~min}$ is considered the minimum for a single event activity, ${ }^{31}$ we determined a single session of PA to be $>10 \mathrm{~min}$. Each frequency $(<1 \times, 1 \times, 2 \times$ and $>3 \times /$ week $)$ of low-intensity, moderate-intensity and vigorous-intensity PA was used in our analyses.

\section{Other variables}

Demographic variables included age, gender, body mass index (BMI), alcohol consumption (never, moderate, heavy and very heavy), smoking status (never, former and current), meat and green/yellow vegetable intake status (never or seldom, once every 2 days and one or more times per day), family history of liver disease (yes or no) and diagnosis and drug usage histories (yes or no) for hypertension, diabetes and dyslipidaemia. A blood sample was drawn from each participant after an overnight fast. The serum triglycerides (TG), low-density lipoprotein cholesterol (LDL-C), high-density lipoprotein cholesterol, fasting plasma glucose (FPG), glycated haemoglobin $(\mathrm{HbAlc})$, aspartate aminotransferase (AST), alanine aminotransferase (ALT) and $\gamma$-glutamyltransferase (GGT) were measured using standard techniques. Systolic blood pressure (SBP) and diastolic blood pressure (DBP) were taken from the right arm using a mercury manometer after the participant rested for at least $15 \mathrm{~min}$ in a sitting position.

\section{Exposure and outcome}

The study's exposure is the PA level at the baseline and the outcome is the future incidence of fatty liver. In never-moderate and heavy alcohol drinkers, incident fatty liver was defined as fatty liver diagnosed by ultrasound.

\section{Statistical analysis}

To compose covariates, we set dichotomous variables (yes or no) for hypertension, diabetes and dyslipidaemia. Hypertension was coded 'yes' if SBP $\geq 140 \mathrm{~mm} \mathrm{Hg}$,
DBP $\geq 90 \mathrm{~mm} \mathrm{Hg}$, there was a diagnosis history or drug usage for hypertension. Diabetes was coded 'yes' if FPG $\geq 7 \mathrm{mmol} / \mathrm{L}, \mathrm{HbAlc} \geq 6.5 \%$, there was a diagnosis history or drug usage for diabetes. Dyslipidaemia was coded 'yes' if LDL-C $\geq 4.1 \mathrm{mmol} / \mathrm{L}, \mathrm{HDL} \leq 1 \mathrm{mmol} / \mathrm{L}$, TG $\geq 2.3 \mathrm{mmol} / \mathrm{L}$, there was a diagnosis history or drug usage for dyslipidaemia.

We performed all analyses on the never-moderate and heavy alcohol drinking groups. To compare baseline characteristics with PA frequencies, we used $\chi^{2}$ tests for categorical variables and analysis of variance for continuous variables. We used the Cox proportional hazard analysis to determine prospective associations between the PA frequency and incident fatty liver. We used two multivariable-adjusted models in this study: covariates of model 1 included age (continuous), gender, BMI (continuous), alcohol consumption (never or moderate for never-moderate alcohol drinkers, and heavy or very heavy for heavy alcohol drinkers), smoking status (never, former or current), family history of liver disease (yes or no), ALT (continuous), GGT (continuous), hypertension (yes or no), diabetes (yes or no), dyslipidaemia (yes or no) and meat and green/yellow vegetable intakes (never or seldom, once every 2 days or one or more times per day). Since there was strong correlation between ALT and AST in both never-moderate $(\mathrm{r}=0.74)$ and heavy alcohol drinkers $(\mathrm{r}=0.76)$, only ALT was entered into the models to avoid multicollinearity; ALT is closely associated with fatty liver. ${ }^{7}$ In model 2 , to consider the effect of PA, we incorporated all three PA intensity variables into model 1 .

We also performed a propensity-adjusted analysis to consider the probability of performing each intensity of PA $>3 \times /$ week $^{32}$ The propensity scores for the highest frequency of the three PA intensities were calculated by a multivariable logistic regression analysis using all covariates. In propensity-adjusted Cox models, we used full samples of $<1 \times$ and $>3 \times /$ week, but did not conduct the matching analysis. ${ }^{32}$ The areas under the receiver operating curves of propensity scores were $0.69-0.76$, respectively. In all Cox models, we integrated the different hazards for baseline starting years using stratification adjustment. The level of significance for all analyses was set at $\mathrm{p}<0.05$. Statistical analyses were performed using SPSS V.21.0 (IBM, Inc, Armonk, New York, USA).

\section{RESULTS}

\section{Description of the sample}

Table 1 shows the participants' baseline characteristics by PA frequency in never-moderate and heavy alcohol drinkers. The mean age of never-moderate drinkers was $47.8 \pm 10.9$ years with men representing $39.5 \%$ of this group. The heavy drinkers' mean age was $49.1 \pm 9.8$ years with $79.6 \%$ men. In both groups, almost half the people did not engage in any PA. Baseline characteristics for all three intensities of PA are presented in online supplementary tables S1a-c. 
Table 1 Baseline characteristics of participants by frequency of physical activity

\begin{tabular}{|c|c|c|c|c|c|c|c|c|c|c|c|c|c|c|c|c|c|c|}
\hline \multirow[b]{2}{*}{ Baseline variables } & \multicolumn{8}{|c|}{$\begin{array}{l}\text { Never-moderate alcohol drinkers } \\
(n=7803 \text {, mean age }=47.8 \pm 10.9 \text { years, male }=39.5 \%)\end{array}$} & \multirow[b]{2}{*}{ p Value } & \multicolumn{8}{|c|}{$\begin{array}{l}\text { Heavy alcohol drinkers } \\
(\mathrm{n}=2343, \text { mean age }=49.1 \pm 9.8 \text { years, male }=79.6 \%) \\
\text { Physical activity (times/week) }\end{array}$} & \multirow[b]{2}{*}{ p Value } \\
\hline & $<1 x$ & & $1 x$ & & $2 x$ & & \multicolumn{2}{|l|}{$\geq 3 x$} & & \multicolumn{2}{|l|}{$<1 x$} & \multicolumn{2}{|l|}{$1 x$} & \multicolumn{2}{|l|}{$2 x$} & \multicolumn{2}{|l|}{$\geq 3 x$} & \\
\hline Number of participants & 3653 & & 1018 & & 816 & & 2316 & & & 1129 & & 322 & & 269 & & 623 & & \\
\hline Mean (SD) age (years) & 45.4 & $(9.9)$ & 46.9 & $(10.6)$ & 49.5 & $(10.7)$ & 51.3 & $(11.5)$ & $<0.001$ & 47.1 & $(9.1)$ & 48.7 & $(9.1)$ & 49.8 & $(9.8)$ & 52.6 & $(10.5)$ & $<0.001$ \\
\hline Male gender & 1494 & $(40.9)$ & 416 & $(40.9)$ & 328 & $(40.2)$ & 842 & (36.4) & 0.004 & 879 & (77.9) & 269 & (83.5) & 224 & (83.3) & 492 & $(79.0)$ & 0.056 \\
\hline Mean (SD) BMI $\left(\mathrm{kg} / \mathrm{m}^{2}\right)$ & 21.6 & $(2.7)$ & 21.6 & $(2.5)$ & 21.6 & $(2.4)$ & 21.7 & (2.6) & 0.485 & 22.4 & $(2.5)$ & 22.9 & $(2.5)$ & 22.7 & $(2.2)$ & 22.6 & (2.3) & 0.017 \\
\hline \multicolumn{19}{|l|}{ Daily alcohol consumption } \\
\hline Never & 638 & $(17.5)$ & 168 & $(16.5)$ & 150 & $(18.4)$ & 460 & (19.9) & 0.055 & - & & - & & - & & - & & \\
\hline Low-mc & 3015 & (82.5) & 850 & (83.5) & 666 & (81.6) & 1856 & $(80.1)$ & & - & & - & & - & & - & & \\
\hline Heavy $(23.0-45.9 \mathrm{~g})$ & - & & - & & - & & - & & & 856 & $(75.8)$ & 254 & (78.9) & 193 & $(71.7)$ & 472 & $(75.8)$ & 0.254 \\
\hline Very heavy $(\geq 46.0 \mathrm{~g})$ & - & & - & & - & & - & & & 273 & (24.2) & 68 & $(21.1)$ & 76 & (28.3) & 151 & (24.2) & \\
\hline Smoking status & & & & & & & & & $<0.001$ & & & & & & & & & 01 \\
\hline Never & 2070 & $(56.7)$ & 651 & $(63.9)$ & 508 & (62.3) & 1502 & $(64.9)$ & & 263 & (23.3) & 72 & (22.4) & 64 & $(23.8)$ & 173 & $(27.8)$ & \\
\hline Former & 620 & $(17.0)$ & 230 & (22.6) & 178 & $(21.8)$ & 539 & (23.3) & & 308 & (27.3) & 119 & $(37.0)$ & 117 & (43.5) & 280 & (44.9) & \\
\hline Current & 963 & (26.4) & 137 & (13.5) & 130 & (15.9) & 275 & (11.9) & & 558 & $(49.4)$ & 131 & $(40.7)$ & 88 & (32.7) & 170 & (27.3) & \\
\hline $\begin{array}{l}\text { Family history of hepatic } \\
\text { disease }\end{array}$ & 203 & (5.6) & 59 & $(5.8)$ & 52 & $(6.4)$ & 144 & (6.2) & 0.674 & 69 & $(6.1)$ & 13 & $(4.0)$ & 22 & (8.2) & 41 & (6.6) & 0.205 \\
\hline Mean (SD) ALT (Units/L) & 19.0 & $(10.4)$ & 18.9 & (9.9) & 18.9 & (8.4) & 18.7 & (8.6) & 0.749 & 22.1 & $(12.6)$ & 22.6 & $(11.5)$ & 22.2 & $(11.2)$ & 21.4 & $(12.6)$ & 0.526 \\
\hline Mean (SD) AST ( & 20.1 & $(5.8)$ & 20.6 & (6.0) & 21.1 & (5.2) & 21.3 & (5.7) & $<0.001$ & 23.1 & (8.9) & 23.7 & $(7.1)$ & 23.5 & $(6.5)$ & 23.7 & (7.9) & 0.484 \\
\hline D) GGT (Units/L) & 27.6 & (25.2) & 28.1 & (33.5) & 27.9 & (24.6) & 26.9 & (23.2) & 0.516 & 62.1 & $(74.1)$ & 58.5 & $(54.3)$ & 59.0 & $(56.1)$ & 53.4 & (52.8) & 0.063 \\
\hline Hypertension* ${ }^{*}$ & 288 & (7.9) & 78 & $(7.7)$ & 110 & (13.5) & 315 & (13.6) & $<0.001$ & 183 & (16.2) & 55 & (17.1) & 63 & (23.4) & 156 & $(25.0)$ & $<0.001$ \\
\hline Diabetes† & 76 & (2.1) & 28 & (2.8) & 35 & $(4.3)$ & 120 & $(5.2)$ & $<0.001$ & 42 & $(3.7)$ & 17 & $(5.3)$ & 21 & $(7.8)$ & 52 & (8.3) & $<0.001$ \\
\hline Dyslipidaemiał & 705 & (19.3) & 220 & $(21.6)$ & 174 & (21.3) & 540 & (23.3) & 0.003 & 236 & (20.9) & 78 & $(24.2)$ & 51 & $(19.0)$ & 142 & (22.8) & 0.354 \\
\hline Meat intake & & & & & & & & & $<0.001$ & & & & & & & & & 0.092 \\
\hline Never or seldo & 1394 & $(38.2)$ & 396 & $(38.9)$ & 348 & $(42.6)$ & 1043 & $(4 !$ & & 469 & $(41.5)$ & 110 & $(34.2)$ & 114 & $(42.4)$ & 280 & $(44.9)$ & \\
\hline Once per 2 days & 1211 & (33.2) & 333 & (32.7) & 260 & (31.9) & 700 & (30.2) & & 356 & (31.5) & 119 & (37.0) & 82 & (30.5) & 182 & (29.2) & \\
\hline Once a day or more & 1048 & (28.7) & 289 & (28.4) & 208 & (25.5) & 573 & $(24.7)$ & & 304 & (26.9) & 93 & (28.9) & 73 & (27.1) & 161 & $(25.8)$ & \\
\hline Vegetak & & & & & & & & & $<0.001$ & & & & & & & & & $<0.001$ \\
\hline Never or seldom & 949 & $(26.0)$ & 163 & $(16.0)$ & 125 & $(15.3)$ & 294 & $(12.7)$ & & 387 & (34.3) & 76 & (23.6) & 63 & (23.4) & 109 & $(17.5)$ & \\
\hline Once per 2 days & 850 & (23.3) & 231 & (22.7) & 157 & (19.2) & 350 & (15.1) & & 297 & (26.3) & 79 & (24.5) & 60 & (22.3) & 123 & (19.7) & \\
\hline Once a day or more & 1854 & (50.8) & 624 & (61.3) & 534 & $(65.4)$ & 1672 & (72.2) & & 445 & (39.4) & 167 & (51.9) & 146 & (54.3) & 391 & (62.8) & \\
\hline \multicolumn{19}{|c|}{$\begin{array}{l}\text { Values are numbers (percentages) unless stated otherwise. } \\
\text { Baseline characteristics for all three intensities of physical activity are presented in online supplementary tables } S 1 \mathrm{a}-\mathrm{c} \text {. } \\
\text { *Systolic blood pressure } \geq 140 \mathrm{~mm} \mathrm{Hg} \text {, diastolic blood pressure } \geq 90 \mathrm{~mm} \mathrm{Hg} \text {, diagnosis history or drug usage for hypertension. } \\
\text { tFasting plasma glucose } \geq 7 \mathrm{mmol} / \mathrm{L}, \mathrm{HbA} 1 \mathrm{c} \geq 6.5 \% \text {, diagnosis history or drug usage for diabetes. } \\
\text { fLow-density lipoprotein cholesterol } \geq 4.1 \mathrm{mmol} / \mathrm{L} \text {, high-density lipoprotein cholesterol } \leq 1 \mathrm{mmol} / \mathrm{L} \text {, serum triglycerides } \geq 2.3 \mathrm{mmol} / \mathrm{L} \text {, diagnosis history or drug usage for dyslipidaemia. } \\
\text { ALT, alanine aminotransferase; AST, aspartate aminotransferase; BMI, body mass index; GGT, } \gamma \text {-glutamyltransferase; HbA1c, glycated haemoglobin. }\end{array}$} \\
\hline
\end{tabular}


During a mean follow-up of 4.4 years (34 648 personyears), 1255 of 7803 never-moderate alcohol drinkers ( $16.1 \%$ of total, $24.9 \%$ of men, $10.4 \%$ of women) developed fatty liver; 520 of 2343 heavy alcohol drinkers (22.2\% of total, $25.4 \%$ of men, $9.6 \%$ of women) developed fatty liver during a mean follow-up of 4.1 years (9596 person-years). In total, 1775 of 10146 participants (17.5\% of total, $25.1 \%$ of men, $10.3 \%$ of women) were newly diagnosed with fatty liver during a mean follow-up of 4.4 years (44 244 person-years).

\section{Incident fatty liver and PA in never-moderate alcohol drinkers}

Table 2 summarises the Cox models in never-moderate alcohol drinkers. In model 2, participants who engaged in low-intensity $\mathrm{PA} \quad(\mathrm{HR}=0.82,95 \%$ CI 0.71 to 0.96$)$ or moderate-intensity PA (HR $=0.56,95 \%$ CI 0.39 to 0.81 ) $>3 \times /$ week significantly reduced their risks of incident fatty liver, compared with those who engaged in $\mathrm{PA}<1 \times /$ week. When participants engaged in vigorous-intensity $\mathrm{PA}>2 \times /$ week, they decreased their risk of fatty liver by about half $(2 \times /$ week: $\mathrm{HR}=0.57,95 \%$ CI 0.38 to 0.86 ; $>3 \times$ : $\mathrm{HR}=0.55$, $95 \%$ CI 0.38 to 0.79 ). All HRs in model 2, including covariates, are shown in online supplementary table S2. The final propensity-adjusted Cox models (see online supplementary table S3) also confirmed the significant preventive effects of $>3 \times$ /week of lower-intensity $(\mathrm{HR}=0.82$, $95 \%$ CI 0.71 to 0.96$)$, moderate-intensity ( $\mathrm{HR}=0.57,95 \%$ CI 0.40 to 0.83 ) and vigorous-intensity PA (HR=0.55, 95\% CI 0.38 to 0.79 ) on fatty liver.

\section{Incident fatty liver and PA in heavy alcohol drinkers}

There were no significant associations between the type or frequency of PA and the risk of incident fatty liver in heavy alcohol drinkers (table 3 ).

\section{DISCUSSION}

This prospective study investigated the association between PA engagement and incident fatty liver in two populations, those with never-moderate or heavy alcohol consumption. We found that PA had an independent effect against incident fatty liver in never-moderate alcohol drinkers, whereas there was no association in heavy alcohol drinkers. Our results suggest that PA is an effective tool for preventing NAFLD as well as other obesity-related diseases. ${ }^{10-12}$

Table 2 HRs of incident fatty liver by frequency of physical activity in never-moderate alcohol drinkers

\begin{tabular}{|c|c|c|c|c|}
\hline & \multicolumn{4}{|c|}{ Frequency of engaging in physical activity (times/week) } \\
\hline & \multirow[b]{2}{*}{$<1 \times$} & \multicolumn{3}{|l|}{ HR $(95 \% \mathrm{Cl})$} \\
\hline & & $1 \times$ & $2 x$ & $\geq 3 \times$ \\
\hline \multicolumn{5}{|l|}{ Low-intensity physical activity } \\
\hline Number of participants & 4900 & 728 & 516 & 1659 \\
\hline Number of person-years & 21679 & 3278 & 2269 & 7422 \\
\hline Number of fatty liver cases & 804 & 108 & 88 & 255 \\
\hline Incidence rates per 1000 person-years & 37 & 33 & 39 & 34 \\
\hline Unadjusted & 1.00 & $0.89(0.73$ to 1.09$)$ & $1.05(0.84$ to 1.31$)$ & $0.93(0.81$ to 1.07$)$ \\
\hline Adjusted for age and gender & 1.00 & $0.87(0.71$ to 1.07$)$ & 0.98 (0.78 to 1.22$)$ & 0.86 (0.74 to 0.99$)$ \\
\hline Model $1^{*}$ & 1.00 & $0.95(0.78$ to 1.17$)$ & 1.00 (0.80 to 1.24$)$ & 0.87 (0.75 to 1.01$)$ \\
\hline Model 2† & 1.00 & $0.91(0.74$ to 1.12$)$ & $0.96(0.77$ to 1.20$)$ & $0.82(0.71$ to 0.96$)$ \\
\hline \multicolumn{5}{|l|}{ Moderate-intensity physical activity } \\
\hline Number of participants & 6699 & 478 & 318 & 308 \\
\hline Number of person-years & 29579 & 2200 & 1441 & 1428 \\
\hline Number of fatty liver cases & 1117 & 67 & 41 & 30 \\
\hline Incidence rates per 1000 person-years & 38 & 30 & 28 & 21 \\
\hline Unadjusted & 1.00 & $0.81(0.63$ to 1.04$)$ & $0.76(0.55$ to 1.03$)$ & 0.56 (0.39 to 0.81$)$ \\
\hline Adjusted for age and gender & 1.00 & 0.81 (0.63 to 1.03$)$ & 0.71 (0.52 to 0.97$)$ & 0.52 (0.36 to 0.75$)$ \\
\hline Model $1^{\star}$ & 1.00 & 0.88 (0.69 to 1.13$)$ & $0.74(0.54$ to 1.01$)$ & $0.56(0.39$ to 0.81$)$ \\
\hline Model 2† & 1.00 & 0.87 (0.68 to 1.12$)$ & $0.74(0.54$ to 1.01$)$ & $0.56(0.39$ to 0.81$)$ \\
\hline \multicolumn{5}{|l|}{ Vigorous-intensity physical activity } \\
\hline Number of participants & 6935 & 328 & 254 & 286 \\
\hline Number of person-years & 30641 & 1484 & 1181 & 1342 \\
\hline Number of fatty liver cases & 1153 & 48 & 24 & 30 \\
\hline Incidence rates per 1000 person-years & 38 & 32 & 20 & 22 \\
\hline Unadjusted & 1.00 & $0.86(0.64$ to 1.15$)$ & 0.54 (0.36 to 0.82$)$ & $0.60(0.42$ to 0.86$)$ \\
\hline Adjusted for age and gender & 1.00 & $0.84(0.63$ to 1.12$)$ & 0.54 (0.36 to 0.82$)$ & 0.55 (0.38 to 0.79$)$ \\
\hline Model $1^{*}$ & 1.00 & $0.87(0.65$ to 1.16$)$ & 0.58 (0.39 to 0.88$)$ & 0.54 (0.38 to 0.78$)$ \\
\hline Model 2† & 1.00 & $0.86(0.64$ to 1.15$)$ & $0.57(0.38$ to 0.86$)$ & 0.55 (0.38 to 0.79$)$ \\
\hline
\end{tabular}


Table 3 HRs of incident fatty liver by frequency of physical activity in heavy alcohol drinkers

\begin{tabular}{|c|c|c|c|c|}
\hline & \multicolumn{4}{|c|}{ Frequency of engaging in physical activity (times/week) } \\
\hline & \multirow[b]{2}{*}{$<1 x$} & \multicolumn{3}{|l|}{ HR (95\% Cl) } \\
\hline & & $1 \times$ & $2 x$ & $\geq 3 \times$ \\
\hline \multicolumn{5}{|l|}{ Low-intensity physical activity } \\
\hline Number of participants & 1554 & 230 & 142 & 417 \\
\hline Number of person-years & 6412 & 901 & 597 & 1686 \\
\hline Number of fatty liver cases & 338 & 47 & 33 & 102 \\
\hline Incidence rates per 1000 person-years & 53 & 52 & 55 & 60 \\
\hline Unadjusted & 1.00 & $0.98(0.72$ to 1.33$)$ & $1.07(0.75$ to 1.53$)$ & $1.14(0.91$ to 1.42$)$ \\
\hline Adjusted for age and gender & 1.00 & $0.93(0.69$ to 1.27$)$ & $1.03(0.72$ to 1.47$)$ & $1.09(0.87$ to 1.37$)$ \\
\hline Model $1^{*}$ & 1.00 & $0.97(0.71$ to 1.32$)$ & 0.97 (0.68 to 1.40$)$ & $1.18(0.93$ to 1.49$)$ \\
\hline Model $2 \dagger$ & 1.00 & 0.98 (0.72 to 1.33$)$ & 0.96 (0.67 to 1.38$)$ & $1.18(0.93$ to 1.50$)$ \\
\hline \multicolumn{5}{|l|}{ Moderate-intensity physical activity } \\
\hline Number of participants & 2002 & 154 & 101 & 86 \\
\hline Number of person-years & 8149 & 666 & 457 & 324 \\
\hline Number of fatty liver cases & 442 & 30 & 27 & 21 \\
\hline Incidence rates per 1000 person-years & 54 & 45 & 59 & 65 \\
\hline Unadjusted & 1.00 & $0.83(0.58$ to 1.21$)$ & $1.09(0.74$ to 1.61$)$ & $1.17(0.75$ to 1.81$)$ \\
\hline Adjusted for age and gender & 1.00 & $0.81(0.56$ to 1.17$)$ & $1.02(0.69$ to 1.50$)$ & $1.05(0.68$ to 1.64$)$ \\
\hline Model $1^{*}$ & 1.00 & $0.82(0.56$ to 1.18$)$ & 1.14 (0.77 to 1.69$)$ & $1.06(0.68$ to 1.66$)$ \\
\hline Model $2 \dagger$ & 1.00 & 0.81 (0.56 to 1.18$)$ & $1.15(0.77$ to 1.71$)$ & $1.13(0.72$ to 1.78$)$ \\
\hline \multicolumn{5}{|l|}{ Vigorous-intensity physical activity } \\
\hline Number of participants & 2055 & 115 & 77 & 96 \\
\hline Number of person-years & 8377 & 488 & 312 & 419 \\
\hline Number of fatty liver cases & 456 & 24 & 21 & 19 \\
\hline Incidence rates per 1000 person-years & 54 & 49 & 67 & 45 \\
\hline Unadjusted & 1.00 & $0.91(0.61$ to 1.38$)$ & $1.20(0.78$ to 1.86$)$ & $0.82(0.52$ to 1.31$)$ \\
\hline Adjusted for age and gender & 1.00 & $0.92(0.61$ to 1.39$)$ & $1.25(0.81$ to 1.94$)$ & $0.79(0.50$ to 1.25$)$ \\
\hline Model $1^{*}$ & 1.00 & $0.86(0.56$ to 1.31$)$ & $1.27(0.81$ to 1.97$)$ & $0.76(0.47$ to 1.22$)$ \\
\hline Model $2 \dagger$ & 1.00 & $0.88(0.57$ to 1.34$)$ & 1.32 (0.85 to 2.07$)$ & $0.77(0.48$ to 1.25$)$ \\
\hline
\end{tabular}

Previous Chinese ${ }^{33}$ and Korean ${ }^{22}$ cohort studies using ultrasound for diagnosis reported that, after 5 years, $11.6 \%$ and $19.3 \%$ of participants, respectively, developed fatty liver. Similarly, in our study during 6-8 years of follow-up (mean 4.4 years), $17.5 \%$ of participants developed fatty liver, which is a feasible rate for Asian populations.

In the never-moderate alcohol drinkers, engaging in PA significantly reduced incident fatty liver, and the effect increased as the intensity and frequency increased. When participants engaged in PA $>3 \times$ /week, the risks of incident fatty liver decreased significantly regardless of the PA intensity. In particular, those who engaged in moderate-intensity PA $>3 \times /$ week, or vigorous-intensity PA $>2 \times /$ week had decreased HRs. In a retrospective study ${ }^{22}$ engaging in $\mathrm{PA}>3 \times /$ week was associated with a lower prevalence of NAFLD. Our prospective findings confirm that study's results, and in addition, show the advantage of higher intensity levels of PA for preventing NAFLD.

Our results might reflect a dose-response relationship between increasing the total amount of PA and decreasing the risk of incident NAFLD; however they may also reflect a special effect of higher intensity levels of PA on NAFLD prevention. Similar to our current findings, a cross-sectional study using biopsy assessment of nonalcoholic steatohepatitis (NASH) ${ }^{21}$ found a significant association between vigorous-intensity $\mathrm{PA}$ and a lower prevalence of NASH, but this was not true for moderate-intensity PA, which was of a similar intensity to our study's low-intensity PA. Intervention studies on PA intensities and abdominal fat also reported that vigorous-intensity PA more strongly reduced abdominal fat than low-intensity PA, even with the same energy expenditure. $^{34} \quad 35$ Kistler et $a l^{21}$ suggested that vigorous-intensity $\mathrm{PA}$ may be better at preventing NAFLD, because of the effect that PA has on AMP-activated protein kinase (AMP-kinase). The activation of AMP-kinase increases ATP production through fatty acid oxidation and glucose transport, and AMP-kinase is activated by depletion of ATP such as in the case of vigorous-intensity PA. ${ }^{21}{ }^{36}$ We also put forward the possible influence of the liver-brain-adipose neurocircuitry recently discovered by Izumida et al, ${ }^{37}$ whereby depletion of liver glycogen triggers the promotion of fat consumption. Higher intensity PA typically promotes liver glycogen catabolism ${ }^{38} 39$ which may promote fat utilisation via this liver-brain-adipose neurocircuitry. 
A meta-analysis by Keating et $a t^{40}$ on exercise and NAFLD showed that exercise with diet intervention was not more effective in reducing liver fat and enzymes compared with diet alone. However, this meta-analysis could not incorporate the exercise intensity because of the lack of data, ${ }^{40}$ which may hide the independent benefit of exercise on NAFLD. Future intervention studies should consider the exercise intensity in addition to the duration and frequency.

The present study investigated the association between PA and incident fatty liver in a population with a high rate of alcohol consumption. Contrary to nevermoderate alcohol drinkers, in heavy alcohol drinkers, the intensity and frequency of PA did not contribute a protective effect on incident fatty liver. Since positive ${ }^{41} 42$ and negative ${ }^{43} 44$ associations have been reported between alcohol consumption and fatty liver disease, the influence of alcohol on the liver is not yet certain. Although the effect that large amounts of alcohol have on the liver may be the reason that we found no association between PA and incident fatty liver in heavy alcohol drinkers, we did not have the details or data to determine this. Further epidemiological and physiological studies are needed. In heavy alcohol drinkers, increasing BMI, being a smoker and having dyslipidaemia were independent predictors for incident fatty liver (see online supplementary table S2), which is similar to previous reports. ${ }^{1} 151645$ Heavy alcohol drinkers should be especially aware of their weight and smoking habits. Increasing BMI and dyslipidaemia were also independent predictors in never-moderate alcohol drinkers, similar to other studies. ${ }^{13}{ }^{14}$ Hence, avoiding obesity is an important aspect in preventing fatty liver for nevermoderate and heavy alcohol drinkers.

This study is the first to reveal the independent preventive effect of PA on incident NAFLD; its strength lies in its prospective cohort design. Additionally, our large sample size allowed us to show separate HRs according to PA frequencies and intensities which revealed the advantages of higher frequencies and intensities of PA. $\mathrm{PA}$ is a cost-effective and non-invasive prescription for good health ${ }^{31}$; and this study reinforces the importance of PA in the prevention of NAFLD.

There were several limitations in this study. First, although hepatic ultrasonography is widely used at the population level, it can lead to incorrect diagnoses. ${ }^{26}$ More precise diagnosis requires liver biopsy. In addition, using several ultrasonography machines during the study may limit the accuracy of diagnoses. However, we believe that this did not seriously affect our results because (1) the similar fatty liver rates obtained at all annual surveys support the reliability of ultrasound diagnosis in the check-ups and (2) all participants randomly/equally shared this error. Second, we did not measure inflammation (eg, serum iron and ferritin) and fibrosis markers (eg, hyaluronic acid and type IV collagen) ${ }^{3}{ }^{3} \mathrm{~A}$ recent intervention study reported that exercise intervention reduced ferritin and thiobarbituric acid reactive substances more than diet therapy in fatty liver patients. ${ }^{46}$ Future research on the effect that PA may have on fatty liver should consider inflammation and fibrosis by measuring these markers and performing biopsies. Third, to maintain an adequate sample size, we did not divide the sample by gender. Women's incidence rate of fatty liver is lower than men's, and alcohol's effect on fatty liver may differ by gender. If we could obtain an adequate sample size for each gender group, a gender difference might be observed. Fourth, because the PA frequency in our questionnaire only went as high as ' $>3 \times /$ week', it was difficult to gauge the total amount of PA at the upper end. Although a more detailed questionnaire would help with this problem, to omit recall bias inherent with self-reported assessments, an objective assessment, such as an accelerometer, is required. Fifth, we focused only on the levels of PA and alcohol consumption at the baseline; the study did not examine the possibility of changing the pattern of PA and alcohol consumption during a follow-up period. To be sure of the effect of PA on fatty liver in never-moderate and heavy drinkers, an intervention study is needed. Sixth, we cannot deny the influence of selection bias; the majority of participants were employees and their spouses in Tokyo, and they might have a higher social status than a rural population. Thus, we may not be able to generalise our findings. The lack of socioeconomic variables such as education and income was also a weakness of the study. Finally, the sample size for heavy drinkers might be inadequate. Although there was no significance, people engaging in $>3 \times /$ week of vigorous-intensity PA were likely to have a lower incident risk of fatty liver, but we cannot determine if this trend reflects the effect of vigorous-intensity PA or just chance with our current data. A larger sample size of heavy alcohol drinkers is needed.

\section{CONCLUSIONS}

This study investigated whether PA reduces the future risk of incident fatty liver in people with never-moderate or heavy alcohol consumption. In never-moderate alcohol drinkers, PA independently reduced the future risk of fatty liver, and HRs decreased as the PA intensity and frequency increased. In contrast, the type or frequency of PA was not significantly associated with incident fatty liver in heavy alcohol drinkers.

PA is a novel tool for preventing NAFLD, along with its well-known effect on other obesity-related diseases. Our prospective cohort findings on fatty liver are currently limited, and more prospective studies are needed to build sound evidence.

Acknowledgements The authors thank the staff at Meiji Yasuda Shinjuku Medical Center for their administrative and practical assistance to the project.

Contributors KT and YK conceived and designed the study, analysed and interpreted the data, and drafted the manuscript. KU and TK acquired and interpreted the data and critically revised the manuscript. TN interpreted the 
data, critically revised the manuscript, and supervised and coordinated the study. All the authors read and approved the final manuscript.

Funding This research received no specific grant from any funding agency in the public, commercial or not-for-profit sectors.

\section{Competing interests None.}

Ethics approval This study was approved by the Ethical Committee of Meij Yasuda Life Foundation of Health and Welfare.

Provenance and peer review Not commissioned; externally peer reviewed.

Data sharing statement No additional data are available.

Open Access This is an Open Access article distributed in accordance with the Creative Commons Attribution Non Commercial (CC BY-NC 4.0) license, which permits others to distribute, remix, adapt, build upon this work noncommercially, and license their derivative works on different terms, provided the original work is properly cited and the use is non-commercial. See: http:// creativecommons.org/licenses/by-nc/4.0/

\section{REFERENCES}

1. Mandayam S, Jamal MM, Morgan TR. Epidemiology of alcoholic liver disease. Semin Liver Dis 2004:24:217-32.

2. McCullough AJ, O'Connor JF. Alcoholic liver disease: proposed recommendations for the American College of Gastroenterology. Am J Gastroenterol 1998;93:2022-36.

3. Farrell GC, Larter CZ. Nonalcoholic fatty liver disease: from steatosis to cirrhosis. Hepatology 2006;43(2 Suppl 1):S99-S112.

4. Angulo P. Nonalcoholic fatty liver disease. N Engl J Med 2002;346:1221-31.

5. Clark JM, Diehl AM. Nonalcoholic fatty liver disease: an underrecognized cause of cryptogenic cirrhosis. JAMA 2003;289:3000-4.

6. Okanoue T, Umemura A, Yasui K, et al. Nonalcoholic fatty liver disease and nonalcoholic steatohepatitis in Japan. $J$ Gastroenterol Hepatol 2011;26(Suppl 1):153-62.

7. Eguchi $\mathrm{Y}$, Hyogo $\mathrm{H}$, Ono $\mathrm{M}$, et al. Prevalence and associated metabolic factors of nonalcoholic fatty liver disease in the general population from 2009 to 2010 in Japan: a multicenter large retrospective study. J Gastroenterol 2012;47:586-95.

8. Lazo M, Clark JM. The epidemiology of nonalcoholic fatty liver disease: a global perspective. Semin Liver Dis 2008;28:339-50.

9. Wong VW. Nonalcoholic fatty liver disease in Asia: a story of growth $J$ Gastroenterol Hepatol 2013;28:18-23.

10. Huai $\mathrm{P}, \mathrm{Xun} \mathrm{H}$, Reilly $\mathrm{KH}$, et al. Physical activity and risk of hypertension: a meta-analysis of prospective cohort studies. Hypertension 2013;62:1021-6.

11. Sigal RJ, Kenny GP, Wasserman DH, et al. Physical activity/ exercise and type 2 diabetes: a consensus statement from the American Diabetes Association. Diabetes Care 2006;29:1433-8.

12. Pattyn N, Cornelissen VA, Eshghi SR, et al. The effect of exercise on the cardiovascular risk factors constituting the metabolic syndrome: a meta-analysis of controlled trials. Sports Med 2013;43:121-33.

13. Fabbrini $E$, Sullivan $S$, Klein $S$. Obesity and nonalcoholic fatty liver disease: biochemical, metabolic, and clinical implications. Hepatology 2010;51:679-89.

14. Hamaguchi M, Kojima T, Takeda N, et al. The metabolic syndrome as a predictor of nonalcoholic fatty liver disease. Ann Intern Med 2005;143:722-8.

15. Naveau S, Giraud V, Borotto E, et al. Excess weight risk factor for alcoholic liver disease. Hepatology 1997;25:108-11.

16. Raynard B, Balian A, Fallik D, et al. Risk factors of fibrosis in alcohol-induced liver disease. Hepatology 2002;35:635-8.

17. Bae JC, Suh S, Park SE, et al. Regular exercise is associated with a reduction in the risk of NAFLD and decreased liver enzymes in individuals with NAFLD independent of obesity in Korean adults. PLOS ONE 2012;7:e46819.

18. Gerber L, Otgonsuren M, Mishra A, et al. Non-alcoholic fatty live disease (NAFLD) is associated with low level of physical activity: a population-based study. Aliment Pharmacol Ther 2012;36:772-81.

19. Krasnoff JB, Painter PL, Wallace JP, et al. Health-related fitness and physical activity in patients with nonalcoholic fatty liver disease. Hepatology 2008:47:1158-66.

20. Zelber-Sagi S, Nitzan-Kaluski D, Goldsmith R, et al. Role of leisure-time physical activity in nonalcoholic fatty liver disease: a population-based study. Hepatology 2008;48:1791-8.
21. Kistler KD, Brunt EM, Clark JM, et al. Physical activity recommendations, exercise intensity, and histological severity of nonalcoholic fatty liver disease. Am J Gastroenterol 2011;106:460-8; quiz 69.

22. Kim HK, Park JY, Lee KU, et al. Effect of body weight and lifestyle changes on long-term course of nonalcoholic fatty liver disease in Koreans. Am J Med Sci 2009;337:98-102.

23. Rector RS, Thyfault JP. Does physical inactivity cause nonalcoholic fatty liver disease? J Appl Physiol (1985) 2011:111:1828-35.

24. Hamaguchi M, Kojima T, Takeda N, et al. Nonalcoholic fatty liver disease is a novel predictor of cardiovascular disease. World $J$ Gastroenterol 2007;13:1579-84.

25. Yajima Y, Ohta K, Narui T, et al. Ultrasonographical diagnosis of fatty liver: significance of the liver-kidney contrast. Tohoku J Exp Med 1983;139:43-50.

26. Hernaez R, Lazo M, Bonekamp S, et al. Diagnostic accuracy and reliability of ultrasonography for the detection of fatty liver: a meta-analysis. Hepatology 2011;54:1082-90.

27. Neuschwander-Tetri BA, Caldwell SH. Nonalcoholic steatohepatitis: summary of an AASLD Single Topic Conference. Hepatology 2003;37:1202-19.

28. Shimazu T, Sasazuki S, Wakai K, et al. Alcohol drinking and primary liver cancer: a pooled analysis of four Japanese cohort studies. Int $J$ Cancer 2012;130:2645-53.

29. Ainsworth BE, Haskell WL, Herrmann SD, et al. 2011 Compendium of Physical Activities: a second update of codes and MET values. Med Sci Sports Exerc 2011;43:1575-81.

30. Ainsworth BE, Haskell WL, Whitt MC, et al. Compendium of physical activities: an update of activity codes and MET intensities. Med Sci Sports Exerc 2000;32(9 Suppl):S498-504.

31. World health organization. Global recommendations on physical activity for health. 2010. http://www.who.int/dietphysicalactivity/ factsheet recommendations/en/

32. Sturmer T, Joshi M, Glynn RJ, et al. A review of the application of propensity score methods yielded increasing use, advantages in specific settings, but not substantially different estimates compared with conventional multivariable methods. J Clin Epidemiol 2006;59:437-47.

33. Wang Y, Li YY, Nie YQ, et al. Association between metabolic syndrome and the development of non-alcoholic fatty liver disease. Exp Ther Med 2013;6:77-84.

34. Irving BA, Davis CK, Brock DW, et al. Effect of exercise training intensity on abdominal visceral fat and body composition. Med Sci Sports Exerc 2008:40:1863-72.

35. Irving BA, Weltman JY, Patrie JT, et al. Effects of exercise training intensity on nocturnal growth hormone secretion in obese adults with the metabolic syndrome. J Clin Endocrinol Metab 2009;94: 1979-86.

36. Kemp BE, Mitchelhill KI, Stapleton D, et al. Dealing with energy demand: the AMP-activated protein kinase. Trends Biochem Sci 1999;24:22-5.

37. Izumida Y, Yahagi N, Takeuchi Y, et al. Glycogen shortage during fasting triggers liver-brain-adipose neurocircuitry to facilitate fat utilization. Nat Commun 2013;4:2316.

38. Hultman E, Greenhaff PL. Carbohydrate metabolism in exercise. In: Maughan RJ, ed. Nutrition in sport. Oxford: Blackwell Science Ltd, 2000:85-96.

39. Casey A, Mann R, Banister K, et al. Effect of carbohydrate ingestion on glycogen resynthesis in human liver and skeletal muscle, measured by (13)C MRS. Am J Physiol Endocrinol Metab 2000;278: E65-75.

40. Keating SE, Hackett DA, George J, et al. Exercise and non-alcoholic fatty liver disease: a systematic review and meta-analysis. J Hepatol 2012;57:157-66.

41. Moriya A, Iwasaki Y, Ohguchi S, et al. Alcohol consumption appears to protect against non-alcoholic fatty liver disease. Aliment Pharmacol Ther 2011;33:378-88.

42. Yamada T, Fukatsu M, Suzuki S, et al. Alcohol drinking may not be a major risk factor for fatty liver in Japanese undergoing a health checkup. Dig Dis Sci 2010;55:176-82.

43. Ekstedt M, Franzen LE, Holmqvist M, et al. Alcohol consumption is associated with progression of hepatic fibrosis in non-alcoholic fatty liver disease. Scand J Gastroenterol 2009;44:366-74.

44. Becker U, Deis A, Sorensen TI, et al. Prediction of risk of liver disease by alcohol intake, sex, and age: a prospective population study. Hepatology 1996;23:1025-9.

45. Klatsky AL, Armstrong MA. Alcohol, smoking, coffee, and cirrhosis Am J Epidemiol 1992;136:1248-57.

46. Oh S, Tanaka $\mathrm{K}$, Warabi $\mathrm{E}$, et al. Exercise reduces inflammation and oxidative stress in obesity-related liver diseases. Med Sci Sports Exerc 2013;45:2214-22. 


\section{Correction}

Tsunoda K, Kai Y, Uchida K, et al. Physical activity and risk of fatty liver in people with different levels of alcohol consumption: a prospective cohort study. BMJ Open 2014;4: e005824. There are three corrections in this paper. These corrections do not change any results or conclusions of the paper.

1. Throughout the paper, the frequencies of physical activity, ' $>2 x /$ week' and ' $>3 x /$ week', should be corrected to ' $\geq 2 \mathrm{x} /$ week' and ' $\geq 3 \mathrm{x} /$ week', respectively.

2. In Table 2 , the entry on the line for Model 1 of Moderate-intensity physical activity in the $2 \mathrm{x}$ column $(0.74$ (0.54 to 1.01$))$ should not be italicised.

3. Table 2 lists aspartate aminotransferase as an adjustment variable. However, as mentioned in the statistical methods, the correct hazard models do not include aspartate aminotransferase, so it should be removed from the table's adjustment variables.

BMJ Open 2015;5:e005824corr1. doi:10.1136/bmjopen-2014-005824corr1

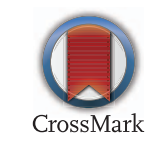

